\title{
Prevention and control of healthcare associated infections within developing countries
}

\author{
Michael A. Borg \\ IFIC Chair, Infection Control Unit, Mater Dei Hospital, Msida, Malta
}

doi: 10.3396/ijic.V6i1.001.10

\begin{abstract}
Many developing countries often face significant health and hygiene challenges that predispose to the transmission of infectious diseases within both community and healthcare settings. Deficient infrastructures, rudimentary equipment and a poor quality of care contribute towards incidences of nosocomial infections which have been estimated to be between 2-6 times higher than those in developed nations. This is the result of varying infrastructural and service deficits within healthcare facilities including inadequate or unsafe water supply, significant overcrowding due to inadequate beds to cope with demand as well lack of strategic direction and planning for healthcare delivery. In order to improve the effectiveness of infection control in many developing countries, a multifactorial set of initiatives needs to be undertaken that are both feasible as well as achievable in the background of economical and social deficits. Hand hygiene is probably the most cost effective of such interventions.

One of the most effective methods for improving infection prevention and control within a country or region is to form an organization in which interested members interact regularly to review practices, share information and support each other. The International Federation of Infection Control (IFIC) provides a forum to link such societies and healthcare professionals working in infection control and related fields worldwide. In this way it is possible to draw on the expertise of its member organizations to network and communicate with each other and assist the development of infrastructure and output within countries that are in early stages of infection control development.
\end{abstract}

\section{Introduction}

Developing countries are normally defined as those lacking the level of nationwide industrialization, infrastructure and technological advances normally found in Western Europe and North America. The vast majority of countries in Africa, Asia, Central \&
South America, Oceania and the Middle East fall in this developing category and often face additional challenges in terms of lower levels of literacy and standards of living. Nevertheless, within this broad group, there are various sub-categories, each having different characteristics as well as economic strengths.

\section{Corresponding author}

Michael A. Borg, IFIC Chair, Infection Control Unit, Mater Dei Hospital, Msida MSD 2090, Malta.

Tel: (+356) 2545 4528, E-mail: michael.a.borg@thelFIC.org 
Indeed some are relatively wealthy oil exporting nations or newly industrializing world economies; a considerable number are middle income countries. At the other end of the development scale lie around fifty very poor nations with predominantly agricultural economies, which tend to be heavily dependent on external aid.

From a medical perspective, many developing countries are often characterised by significant health and hygiene issues. Indeed it has been estimated that more than 1 billion inhabitants in these countries do not have access to safe water and even less to basic sanitation. ${ }^{1}$ Around 1.5 million children in the developing world die per year; diarrhoea is responsible for more than $80 \%$ of these deaths. ${ }^{2}$ One of the reasons for this state of affairs in poorer countries is the low budgetary allocation and expenditure on health. Indeed the proportion of annual expenditure for health related initiatives in many developing countries is often less than $5 \%$ of Gross Domestic Product (GDP), sometimes less than $0.1 \%{ }^{3}$

\section{Healthcare associated infections in developing countries}

Unlike more affluent countries, infectious diseases continue to pose a heavy burden of morbidity as well as mortality in developing nations. ${ }^{4}$ Amongst the more important infections are a wide range of respiratory diseases including tuberculosis, various gastrointestinal infections, AIDS and HIV plus a spate of parasitic infestations of which malaria is the most significant. However this situation is not limited to ambulatory settings and is equally relevant within healthcare institutions. Deficient infrastructures, rudimentary equipment and a poor quality of care contribute towards incidences of nosocomial infections which have been estimated to be between 2-6 times higher than those in developed nations. ${ }^{5}$ In many instances, such figures are often guesstimates because surveillance systems are often non existent or unreliable. However, the limited studies on prevalence of healthcare associated infections in some developing countries suggest that up to $40 \%$ of these are probably preventable. ${ }^{5}$ This situation appears to particularly severe within intensive care settings where up to 60 to 90 infections per 1000 care-days have been reported; excess mortality rates in more severe infections such as blood stream and lower respiratory infections approaches $25 \%$ in adults and more than $50 \%$ in neonates. ${ }^{6}$

Healthcare associated infection in developing countries also involves a wider spectrum of pathogens than is normally seen found in the western world. Numerous publications have highlighted the frequency with which community infections, such as cholera, measles and infectious diarrhoea, spread nosocomially within such institutions. ${ }^{7,8}$ In many instances outbreaks are traceable to an index case which had been inappropriately managed in an environment of overcrowding and limited hospital hygiene. ${ }^{9}$ Tuberculosis transmission in healthcare facilities is common in many African countries as well as parts of Asia and Latin America. ${ }^{10}$ In many instances this is related to the rise of HIV in these regions and may be complicated by increasing prevalence of multi drug resistance. Blood borne infections are not restricted to HIV: hepatitis B virus remains a major nosocomial pathogen in many hospitals within the developing world. ${ }^{11}$ More dramatic and life threatening have been outbreaks of viral haemorrhagic fevers in institutions within several countries in the African continent. ${ }^{12}$ Hospitals in poorer countries are also affected by healthcare associated infections caused by more conventional pathogens which, just like in their western counterparts, can carry the additional burden of antimicrobial resistance. ${ }^{4}$ Data on the prevalence of resistance in nosocomial pathogens in developing countries is poorly documented but recent publications suggest that it may be even more common than in the industrialised West. Recent reports from the Mediterranean region have highlighted methicillin resistance rates in Staphylococcus aureus exceeding $50 \%$ in several countries in the Middle East and resistance to third generation cephalosporins in $E$. coli exceeding $70 \%$ in some participating hospitals. ${ }^{13}$ There may be diverse and often complex backgrounds to this epidemiological situation.

\section{Factors affecting transmission and management of nosocomial infections}

The infrastructure of healthcare facilities in some of the poorer nations often lacks basic requirements for the prevention of transmission of infectious diseases. Inadequate or unsafe water supplies together with lack of resources or equipment for affective environmental cleaning is often compounded by significant 
overcrowding due to inadequate beds to cope with demand. ${ }^{14}$ There is often a lack of strategic direction and effective planning for healthcare delivery at both national and local levels. A functional sterilisation department is by no means standard in every hospital, even in the larger urban institutions. Other areas of concern include poor awareness or knowledge about communicable disease transmission amongst healthcare workers and lack of commitment within senior management. ${ }^{15}$ This is particularly significant in developing countries where nurses, doctors and patients are often unaware of the importance of infection control and its relevance to safe healthcare. ${ }^{16}$ Medical practitioners may have a tendency to be focused on individual patients and disinclined to think of them in groups, a viewpoint which undermines basic principles of infection prevention and control. ${ }^{17}$ They are often unaware of the need to minimise the risks of nosocomial infection, regarding them as natural or inevitable. ${ }^{18} \mathrm{On}$ the other hand, nurses have more intimate contact with patients and are trained to take care of patients in groups. Although this increases their potential to serve as sources of cross-transmission, nurses are likely to have more positive attitudes towards infection control policies. However this is hindered by the comparatively low status of nurses in the developing world and a gender bias in regions where emancipation of women has been slow. Attitudes of senior medical staff may further compound the problem through personality clashes, resistance to change or improvement and reluctance to work in tandem with other health professionals. Non existent litigation further accentuates lack of accountability at various levels. Furthermore, many patients have limited expectations, already regarding themselves fortunate to have any sort of institutional care and as a result accept a significant degree of morbidity as an inevitable part of their hospital stay. It must be emphasised that, even in the poorer countries, this set of circumstances is by no means universal in all hospitals. Even when most hospitals in a country lack basic systems of infection prevention and control, some individual institutions (often either private or NGO managed) have infection control standards of the highest quality. However, only be a small minority of patients, often coming from a more affluent background, can benefit from them. The risks of infection in hospitals within the developing world are not restricted to patients; occupational health has an equally low priority in many of these regions and healthcare workers may be become infected by healthcare associated infections such as viral hepatitis, HIV and tuberculosis.

In these environments with limited resources and where medical practice is biased towards intervention rather than prevention, it is not surprising that basic infection control programmes are often lacking, particularly in smaller hospitals in rural areas. ${ }^{18}$ Even within larger urban facilities, infection control teams, comprising at least one trained infection control nurse and doctor with managerial backup, are very much in the minority. They are often restricted to academic institutions, heavily funded government or private tertiary care units. Even where present, these teams tend to encounter numerous logistical obstacles including lack administrative, clerical and IT support. The quality of infection control is therefore highly variable; policies and procedures are frequently absent or lack an evidence base or local consultation, and often fail to address local needs.

Healthcare professionals also face significant challenges in the diagnosis and treatment of infectious disease. ${ }^{4}$ Diagnostic facilities are often lacking: laboratories may be absent or have inadequate material and human resources. Trained laboratory scientists are very much in the minority and quality control programs to ensure the validity of laboratory results often lacking. As a result, clinicians may lack confidence in the laboratory and prefer to treat blindly, using only on clinical judgement or recommendations from other countries rather than local epidemiology. One reason for this is the lack of feedback of local resistance data. ${ }^{20}$ This risks inappropriate treatment which does not properly cover local resistance prevalence patterns. Another major factor hindering the treatment of infectious disease is the use of poor quality (sometimes counterfeit) antimicrobials that may contain little or no active ingredient within the formulation. ${ }^{21}$

\section{Addressing the challenge}

In order to improve the effectiveness of infection control systems in many developing countries, a multifactorial set of initiatives needs to be implemented, taking account of economic and social realities. ${ }^{15}$ It is essential that infection control raise their profiles within hospitals 
in these regions. These key personnel must be provided with the necessary training, administrative support and facilities in order to deliver the services required. Such teams would be able to identify the major challenges and assess relevant risks through tailored surveillance programmes. Surveillance constitutes a challenge in such environments since it is often time consuming and resource dependent ${ }^{22}$ and requires a reasonable level of laboratory support. Nevertheless, by using the simplified definitions of healthcare associated infections suggested by the World Health Organisation, it is possible to achieve a surveillance programme even with very limited resources. ${ }^{23}$ Such initiatives need to concentrate on the more serious infections and document their impact in the respective facility. Trained infection control personnel can also help eliminate wasteful rituals which siphon resources away from truly effective practices. Dogmas that should be discarded include routine use of disinfectants for environmental cleaning, use of unnecessary personal protective equipment such as overshoes and excessive waste management procedures which treat all waste generated in the hospital as infectious. Infection Control teams can spearhead cost-effective interventions based on training of healthcare workers to comply with standard precautions, patient isolation and occupational health and safety. It is possible to achieve significant reduction in the prevalence of healthcare associated infections through low cost measures; interventions aimed at preventing cross transmission of infection are particularly effective.

There is no doubt that one of the most cost effective interventions in limited resource environments is improved compliance with hand hygiene. The World Health Organisation has designated improvement of health hygiene within healthcare facilities worldwide as a priority and chose this topic for its first Global Patient Safety Challenge under the banner 'Clean Care is Safer Care'. ${ }^{6}$ A comprehensive set of tools have been tested worldwide in pilot hospitals, the majority of which were in developing countries. This initiative focuses on the availability and utilisation of alcohol hand rub for patient contact situations where hands are physically clean. This is made possible through local manufacture of inexpensive, good quality products according to a validated formula. A multimodal strategy requires these alcohol hand rub containers to be available at point of care and for the staff of the hospital to receive adequate training and education in their use. Hand hygiene practices are monitored and feedback on performance regularly provided to the users. Reminders in the workplace increases awareness and belief amongst healthcare workers in general.

\section{The International Federation of Infection Control}

It is clear that to achieve effective improvements in infection prevention and control, healthcare facilities in many developing countries need support and assistance. Experience suggests that one of the most effective and rapid methods for improving infection prevention and control within a country or region is to form an organization in which interested members interact regularly to review practices and share information. ${ }^{21}$ In this way, the more knowledgeable members help those who are learning an all participate in education and sharing experience. This maximizes group skills and reduces the sometimes steep learning curve that is often required. In addition, the organization provides credibility for change and can help develop practice guidelines and official regulations in the field. The International Federation of Infection Control (IFIC) provides a forum to link societies and healthcare professionals working in infection control and related fields worldwide. The goals of the Federation are to provide a communication and support network, draw on the expertise of member organizations to help each other and to assist in the development of infrastructure and output within countries that are in early stages of infection control development. IFIC promotes high quality educational opportunities, including publications, teaching materials and training programs at no or low cost, and conferences and workshops for infection control practitioners from the widest possible resource background and experience. There is close liaison with the World Health Organisation and other organisations that promote infection prevention worldwide, and especially in limited resource settings. In addition to Society Membership, the Federation has recently introduced personal Associate Membership to allow individuals who do not yet have an infection control association in their own country or who would like a more intensive participation in IFIC, to register as individual members so that they can contribute to global infection control initiatives and network with others from all over the world. IFIC Associate membership is free to infection control professionals living and working in more than 150 developing countries. 
The IFIC web site (www.thelFIC.org) provides information about IFIC and its activities and provides information and resources on infection control. It is also a forum for networking with organizations, agencies and IFIC corporate sponsors. The official publication of the Federation is the International Journal of Infection Control, published electronically biannually. The journal provides a forum for education and discussion and publication of research articles and contributions on local infection control challenges and practices. The journal is available in electronic format at www. ijic.info. It is free access and no fee is requested to publish articles. It also offers authors from developing countries, whose first language is usually not English, help with correction or editing of manuscripts. In this way, the journal provides a forum for communication and sharing of data and successful initiatives relevant to infection prevention and control in developing countries. IFIC also publishes an e-newsletter that is also circulated to the membership quarterly.

IFIC's infection control manual, Basic Concepts in Infection Control, is authored by experts on infection control. The intent of the manual is to provide a scientific foundation for basic infection control principles, mainly to countries with limited resources. Past editions of the book have been translated into Albanian, Arabic, Chinese, Croatian, French, Greek, Lithuanian, Persian, Spanish, Thai and Turkish languages. The book is available for download, free of charge, from our website. IFIC has also developed lecture slides based on the second edition of Infection Control: Basic Concepts and Training. Like all educational materials, the PowerPoint presentations are available free of charge and can also be downloaded from the IFIC web site.

IFIC places particular emphasis on improving contacts and dialogue between infection control societies in developed countries with their counterparts in limitedresource settings. One effective way to achieve this is through the organisation of low-cost, high-quality annual congresses, often held in conjunction with a local hosting member society. Over the past years, meetings have held in Santiago, Budapest, S. Africa, Turkey, Croatia, Malta and Egypt. Several scholarships are awarded at each congress to chosen applicants, active in practice of infection control, following submission of a descriptive abstract judged by an independent scientific review committee. Preference is given to participants in developing countries with limited resources. IFIC also reviews, evaluates and supports initiatives consistent with its goals, and assists in bringing them to fruition wherever possible. This includes research or surveillance projects for which help is needed in obtaining grant support from industry and foundations.

\section{Conclusion}

Infection prevention and control in healthcare facilities within the developing world continues to offer numerous challenges as a result of reduced resources related to socio-economics, infrastructure and human resources. However it is possible to achieve substantial progress even within such challenging circumstances through programmes led by trained and empowered infection control professionals. Such initiatives need to concentrate on low cost, high impact interventions with appropriate training and interaction and networking with colleagues and societies within the country itself and beyond. IFIC is one of the key organisations that can help with this.

\section{Acknowledgements}

This paper is adapted, with permission, from the publication: Infection Control in Developing Countries carried in the 2009 special edition of Culture

\section{References}

1. Moe CL, Rheingans RD. Global challenges in water, sanitation and health. J Water Health 2006; 4 Suppl 1: 41-57.

2. Boschi-Pinto C, Velebit L, Shibuya K. Estimating child mortality due to diarrhoea in developing countries. Bull World Health Organ 2008; 86: 710-717.

3. World Health Organization. Implementation of the global strategy for health for all by the year 2000. Eighth report on the world health situation. Volume 6 Eastern Mediterranean Region. Second Evaluation. World Health Organization. Regional Office Eastern Mediterranean Region, Alexandria, Egypt; 1996.

4. Shears P. Poverty and infection in the developing world: healthcare-related infections and infection control in the tropics. J Hosp Infect 2007; 67: 217-224.

5. Wenzel RP. Towards a global perspective of nosocomial infections. Eur / Clin Microbiol 1987; 6: 341-343.

6. Pittet D, Allegranzi B, Storr J et al. Infection control as a major World Health Organization priority for developing countries. J Hosp Infect 2008; 68: 285-292.

7. Mhalu FS, Mtango FD, Msengi AE. Hospital outbreaks of cholera transmitted through close person to person contact. Lancet 1984; ii: 82-84. 
8. Vaagland $\mathrm{H}$, Blomberg B, Kruger C, Naman M, Jureen R, Langeland N. Nosocomial outbreak of neonatal Salmonella enteritidis in a rural hospital in northern Tanzania. BMC Infect Dis 2004; 4: 35.

9. Marshall TM, Hlatswayo D, Schoub B. Nosocomial outbreaks - a potential threat to the elimination of measles? J Infect Dis 2003; 187: S97-S101.

10. Mehtar S. Lowbury Lecture 2007: infection prevention and control strategies for tuberculosis in developing countries lessons learnt from Africa. J Hosp Infect 2008; 69: 321-327.

11. Lynch P, Pittet D, Borg MA, Mehtar S. Infection control in countries with limited resources. J Hosp Infect 2007; 65 Suppl 2: 148-150.

12. Fisher-Hoch SP. Lessons from nosocomial haemhorragic fever outbreaks. Br Med Bull 2005; 73: 123-137.

13. Borg MA, Scicluna E, de Kraker $M$ et al. Antibiotic resistance in the southeastern Mediterranean - preliminary results from the ARMed project. Euro Surveill 2006; 11: 164-167.

14. Borg MA, Cookson BD, Gür D et al. Infection control and antibiotic stewardship practices reported by south-eastern Mediterranean hospitals collaborating in the ARMed project. J Hosp Infect 2008; 70(3): 228-234.

15. Damani N. Simple measures save lives: an approach to infection control in countries with limited resources. J Hosp Infect 2007; 65 Suppl 2: 151-154.
16. Sobayo El. Nursing aspects of infection control in developing countries. J Hosp Infect 1991; 18: 388-391.

17. Meers PD. Infection control in developing countries. J Hosp Infect 1988; 11: 406-410.

18. Ponce-de-Leon S. The needs of developing countries and the resources required. J Hosp Infect 1991; 18: 378-381.

19. Raza MW, Kazi BM, Mustafa M, Gould FK. Developing countries have their own characteristic problems with infection control. J Hosp Infect 2004; 57: 294-299.

20. Borg MA, Cookson BD, Scicluna E, ARMed Project Steering Group and Collaborators. Survey of infection control infrastructure in selected southern and eastern Mediterranean hospitals. Clin Microbiol Infect 2007; 13: 344-346.

21. Lynch $P$, Rosenthal VD, Borg MA, Eremin SR. Infection Control: A Global View in Jarvis WR: Bennett \& Brachman's Hospital Infections; 2007. Lippincott, Williams and Wilkins, Philadelphia.

22. Damani N. Surveillance in Countries with Limited Resources. Int J Infect Contr 2008; doi:10.3396/ijic.V4i1.003.08

23. World Health Organisation. Prevention of hospital acquired infections: A Practical Guide. 2nd ed. Geneva: World Health Organization, 2002. WHO/CDR/EPH/2002.12. 